\title{
Stochastic Signatures of Phase Space Decomposition
}

\author{
John J. Kozak ${ }^{1}$ and Roberto A. Garza-López ${ }^{2}$ \\ ${ }^{1}$ Depaul University, College of Digital Media and Computing, 243 South Wabash Avenue, Chicago, IL 60604-2301, USA \\ ${ }^{2}$ Department of Chemistry and Seaver Chemistry Laboratory, Pomona College, Claremont, CA 91711, USA
}

Correspondence should be addressed to Roberto A. Garza-López, rgarza@pomona.edu

Received 28 July 2011; Accepted 15 September 2011

Academic Editors: M.-B. Hu and O. Kuksenok

Copyright ( 92012 J. J. Kozak and R. A. Garza-López. This is an open access article distributed under the Creative Commons Attribution License, which permits unrestricted use, distribution, and reproduction in any medium, provided the original work is properly cited.

\begin{abstract}
We explore the consequences of metrically decomposing a finite phase space, modeled as a $d$-dimensional lattice, into disjoint subspaces (lattices). Ergodic flows of a test particle undergoing an unbiased random walk are characterized by implementing the theory of finite Markov processes. Insights drawn from number theory are used to design the sublattices, the roles of lattice symmetry and system dimensionality are separately considered, and new lattice invariance relations are derived to corroborate the numerical accuracy of the calculated results. We find that the reaction efficiency in a finite system is strongly dependent not only on whether the system is compartmentalized, but also on whether the overall reaction space of the microreactor is further partitioned into separable reactors. We find that the reaction efficiency in a finite system is strongly dependent not only on whether the system is compartmentalized, but also on whether the overall reaction space of the microreactor is further partitioned into separable reactors. The sensitivity of kinetic processes in nanoassemblies to the dimensionality of compartmentalized reaction spaces is quantified.
\end{abstract}

\section{Introduction}

To provide a physical motivation for the present study, understanding the factors influencing self-assembly in nanophase materials is a major experimental and theoretical challenge $[1,2]$. Modeling even the early stages of selfassembly already introduces fundamental questions, for example, whether the process is reducible to a sequence of elementary steps occurring under equilibrium conditions $[3,4]$. The role of different system geometries in influencing the efficiency of reactions in a finite, compartmentalized system [5] is an ongoing problem, one aspect of which is explored quantitatively here.

Before discussing the technical aspects of our work, we illustrate the main idea with a simple example. Consider a $d=2$ dimensional lattice of $N=100$ sites. One possible representation of such a lattice would be a $10 \times 10$ system (see Figure 1). But, another representation that comprises $N=100$ sites would be the disjoint pair of lattices, a $6 \times 6$ lattice with $N=36$ sites, and an $8 \times 8$ lattice with $N=64$ sites, linked at a common corner site. To monitor ergodic flows on the assigned phase space(s), we place a sink (trap) at one of the corner vertices of the $10 \times 10$ lattice and at the common vertex of the two sublattices. The transit time of a test particle undergoing unbiased, random displacements until, eventually, it is (irreversibly) localized at the trap can be determined by formulating and then solving numerically the stochastic master equation [6] for the problem. By extracting the first moment of the underlying probability distribution function, specified by the set of site-specific mean walklengths $\langle n(i)\rangle$, the overall mean number $\langle n\rangle$ of steps before localization, a signature of the mean transit time, can be obtained.

One fully expects to get different values of $\langle n\rangle$ for these two lattice representations, but the question is "how different?" Also, the problem was illustrated above using (three) $n \times n$ lattices. How do the results change if one or more of the disjoint spaces are $n \times m$ lattices, that is, not approximately "square?" Is the difference in reaction efficiency magnified or suppressed? And, further, do these differences persist if one considers phase spaces of higher $(d=3)$ dimension? It is to the above questions that the present contribution is directed. 


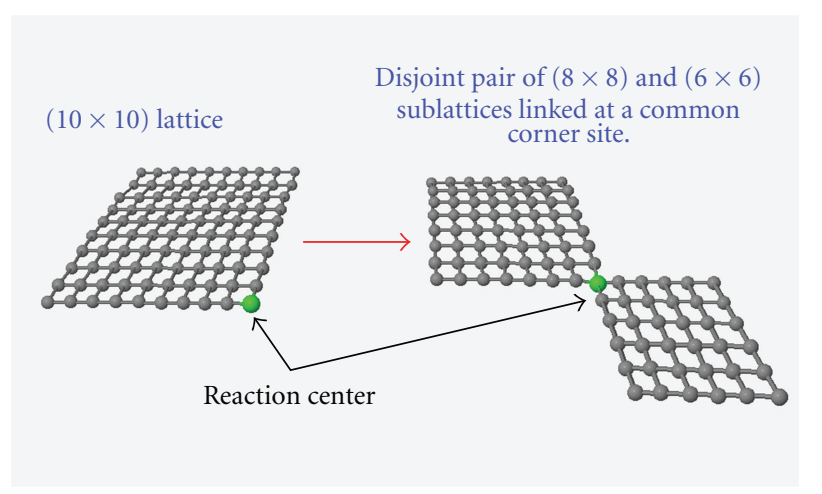

Figure 1: A $d=2$ dimensional lattice of $N=100$ sites. One possible representation of such a lattice would be a $10 \times 10$ system. But, another representation that comprises $N=100$ sites would be the disjoint pair of lattices, a $6 \times 6$ lattice with $N=36$ sites and an $8 \times 8$ lattice with $N=64$ sites, linked at a common corner site (articulation point).

Fundamental results in number theory can be used to motivate the choices of lattices for which the metric decomposability of phase space can be studied. For dimension $d=$ 2, one can take advantage of Fermat's last theorem, namely, that one can find three positive integers $a, b$, and $c$ that satisfy the equation $a^{n}+b^{n}=c^{n}$ for $n=2$ for a given choice of $c^{n}$. In $d=2$, the decomposition is not unique; for any given $c$, there may be (and usually is) more than one choice of $\{a, b\}$ that satisfies the theorem.

In three dimensions, however, the possibility of finding multiple lattices with such a degeneracy is much more limited, in fact, just the set of Hardy-Ramanujan numbers. The first is $2=1^{3}+1^{3}$; the second "taxi cab" number is

$$
1729=1^{3}+12^{3}=9^{3}+10^{3}=7 \times 13 \times 19 .
$$

These lattices are, practically speaking, as far as one can go. The next smallest Hardy-Ramanujan number is

$$
\begin{aligned}
87539319 & =167^{3}+436^{3}=228^{3}+423^{3} \\
& =255^{3}+414^{3}=223 \times 469 \times 837,
\end{aligned}
$$

the last factorization one of several possible. Given the computational demands in implementing the theory of finite Markov processes, these lattices are just too large; hence, our results for dimension $d=3$ presented in Section 3 are (much) more limited than for $d=2$.

The following section specifies the lattice statistical parameters needed to develop the Markovian theory. Section 3 presents the results of a series of calculations for $d=2$ and $d=3$ dimensional lattices. In Section 4 , site-specific walklength data are used to deduce two new invariance relations, valid for finite lattices, which must be satisfied exactly to confirm that the computational results are numerically exact. In the final section, we comment on the relevance of

\begin{tabular}{|c|c|c|c|c|c|c|}
\hline$d$ & Sites & Lattice & $\langle n\rangle$ & Lattice & $\langle n\rangle$ & $\langle n\rangle_{T}$ \\
\hline \multirow[t]{11}{*}{2} & 50 & $1 \times 1$ & 0.0 & $7 \times 7$ & 212.354 & 212.354 \\
\hline & & $5 \times 5$ & 88.690 & $5 \times 5$ & 88.690 & 177.380 \\
\hline & & $5 \times 10$ & & & & 240.537 \\
\hline & 65 & $1 \times 1$ & 0.0 & $8 \times 8$ & 297.846 & 297.846 \\
\hline & & $4 \times 4$ & 48.762 & $7 \times 7$ & 212.354 & 261.116 \\
\hline & & $5 \times 13$ & & & & 363.840 \\
\hline & 85 & $2 \times 2$ & 6.667 & $9 \times 9$ & 400.071 & 406.738 \\
\hline & & $6 \times 6$ & 142.902 & $7 \times 7$ & 212.354 & 355.256 \\
\hline & & $5 \times 17$ & & & & 563.571 \\
\hline & 100 & $6 \times 6$ & 142.902 & $8 \times 8$ & 297.846 & 440.748 \\
\hline & & $10 \times 10$ & & & & 519.637 \\
\hline
\end{tabular}
our results to self-assembly in nanosystems.
TABLE 1: $d=2$ dimensional Lattices.

\section{Formulation}

We consider finite planar lattices, each site of which is of valence (connectivity) $v=4$, and finite $d=3$ dimensional lattices, each site of which is of valence $v=6$. At any interior site of the lattice, the test particle can move with equal a priori probability in any one of $v$ directions. If the particle initiates its motion at an edge site on a $d=2$ lattice, it can move in one of three directions on the lattice, but if it attempts to step "out of" the lattice, it is reset at the starting position. If the particle happens to be at a corner site, it can move to two adjacent lattice sites, remaining on the lattice, or is "reset" (stalls) at the corner site twice, rather than leaving the lattice. A similar protocol is implemented on $d=3$ lattices, where a particle on an interior site can move in $v$ directions, on a face site in $v-1$ directions with one reset, on a edge site in $\nu-2$ directions with two resets, and on a corner site in $v-3$ directions with three resets.

In all calculations here, the sink is placed at one corner site. Previous studies [5] have documented that moving the trap away from a centrosymmetric location will increase the walklength. However, the parent lattice or the lattices that result when one deconstructs a given initial lattice may not have a unique centrosymmetric site; for definiteness, we anchor the trap at a corner site. All calculations were carried out for nearest-neighbor displacements only and in the absence of any governing potentials; both restrictions can be lifted in further work.

All results reported here, Tables 1-3, are numerically exact (see Section 4). Once the problem is formulated, no further approximations are made in carrying out the computational program.

\section{Results}

Displayed in Table 1 are representative results for the $d=$ 2 dimensional case. In every instance, one expects and finds that the results for $\langle n\rangle$ are systematically larger for the "parent" lattice than for the corresponding two disjoint lattices. The more "symmetric" and "similar" are the pair of disjoint lattices, the more efficient is the underlying ergodic process. Computing the percent difference between results 
Table 2: $N=1759$.

\begin{tabular}{|c|c|c|c|c|c|c|c|}
\hline$d$ & Lattice & $\langle n\rangle$ & Lattice & $\langle n\rangle$ & Lattice & $\langle n\rangle$ & $\langle n\rangle_{C}$ \\
\hline \multirow[t]{2}{*}{2} & $5 \times 5$ & 88.690 & $11 \times 11$ & 657.085 & $71 \times 71$ & 51060.819 & $17268.865^{a}$ \\
\hline & $19 \times 91$ & & & & & & 20438.245 \\
\hline \multirow[t]{3}{*}{3} & $1 \times 1 \times 1$ & 0.0 & $12 \times 12 \times 12$ & 6691.079 & & & 6691.079 \\
\hline & $9 \times 9 \times 9$ & 2716.171 & $10 \times 10 \times 10$ & 3784.118 & & & 6500.289 \\
\hline & $7 \times 13 \times 19$ & & & & & & 6939.812 \\
\hline
\end{tabular}

$1729=\left(5^{2}+11^{2}+71^{2}\right) / 3$.

Here, $\langle n\rangle_{C}=\left(\langle n\rangle_{5 \times 5}+\langle n\rangle_{11 \times 11}+\langle n\rangle_{71 \times 71}\right) / 3$.

TABLE 3: Invariance relations.

\begin{tabular}{|c|c|c|c|c|}
\hline$d=2$ & Lattice & $N$ & $\sum(1)$ & $\sum(2)$ \\
\hline & $2 \times 2$ & 4 & 12 & 40 \\
\hline & $4 \times 4$ & 16 & 60 & 232 \\
\hline & $5 \times 5$ & 25 & 96 & 376 \\
\hline & $6 \times 6$ & 36 & 140 & 552 \\
\hline & $7 \times 7$ & 49 & 192 & 760 \\
\hline & $5 \times 10$ & 50 & 196 & 776 \\
\hline & $8 \times 8$ & 64 & 252 & 1000 \\
\hline & $5 \times 13$ & 65 & 256 & 1016 \\
\hline & $9 \times 9$ & 81 & 320 & 1272 \\
\hline & $5 \times 17$ & 85 & 336 & 1336 \\
\hline & $10 \times 10$ & 100 & 396 & 1576 \\
\hline & $11 \times 11$ & 121 & 480 & 1912 \\
\hline & $19 \times 91$ & 1729 & 6912 & 27640 \\
\hline & $71 \times 71$ & 5041 & 20160 & 80632 \\
\hline \multirow[t]{6}{*}{$d=3$} & Lattice & $N$ & $\sum(1)$ & $\sum(2)$ \\
\hline & $3 \times 3 \times 3$ & 27 & 156 & 918 \\
\hline & $9 \times 9 \times 9$ & 729 & 4368 & 26190 \\
\hline & $10 \times 10 \times 10$ & 1000 & 5994 & 35946 \\
\hline & $12 \times 12 \times 12$ & 1728 & 10362 & 62154 \\
\hline & $7 \times 13 \times 19$ & 1729 & 10368 & 62190 \\
\hline
\end{tabular}

for the composite lattice versus two disjoint (but linked) lattices, one finds that the difference is always greater than $10 \%$, and frequently much greater (in the range from $10 \%$ $30 \%$, depending on lattice symmetry).

In Table 2, we find that the difference in results for $d=3$ dimensions is much less severe. For $N=1729$, the percentage is less than $4 \%$. Hence, dimensionality appears to play the critical role in cases studied here. Note that there is a companion, $d=2$ dimensional result in Table 2 which was obtained by taking advantage of the decomposition of $N=$ 1729 into the average of the greatest member in each pair of Brown numbers $(5,4),(11,5)$, and $(71,7)$. Once again, the difference between the (here) three disjoint lattices and the parent $19 \times 91$ lattices is $\sim 15 \%$.

We present in the following section results for two new lattice invariance relations that can be used to check the accuracy of the site-specific $\langle n(i)\rangle$ values that underlie the data given in Tables 1 and 2 .

\section{Invariance Relations}

Over 40 years ago, Montroll and Weiss [7] reported for lattices subject to periodic boundary conditions an exact, analytic result for the average number of steps taken by a random walker from a site which is a first nearest-neighbor to a (deep) trap. Further, they discussed how one would proceed to derive similar analytic expressions starting from sites second, third, ...nth nearest neighbor(s) to the trap, emphasizing that the derived expressions will depend on the structure of the lattice.

In previous work, we pursued the Montroll-Weiss program and obtained additional invariance relations for hexagonal, square planar, and cubic lattices subject to periodic boundary conditions, each having a single, centrosymmetric, deep trap [8-10]. Since satisfaction of lattice invariance relations is an "acid test" on the accuracy of numerical calculations of the site-specific $\langle n(i)\rangle$ (and overall 
mean $\langle n\rangle)$, we used the methods described in [8-10] to develop invariance relations for finite lattices.

The corresponding first Montroll-Weiss invariance relation for finite lattices

$\sum(1) \equiv$ Sum of the $\langle n(i)\rangle$ values

for all sites first nearest-neighbor to the trap

$$
=v(N-1)
$$

holds for a trap positioned either at an interior site or a surface site. The second invariance relation obtained for finite lattices is

$$
\begin{aligned}
\sum(2) \equiv & \text { Sum of the }\langle n(i)\rangle \text { values } \\
& \quad \text { for all sites second nearest neighbor to the trap } \\
= & v[v(N-1)]+2 v
\end{aligned}
$$

for $d=2$ lattices with a trap at a corner site and

$$
\begin{aligned}
\sum(2) \equiv & \text { Sum of the }\langle n(i)\rangle \text { values } \\
& \text { for all sites second nearest neighbor to the trap } \\
= & v[v(N-1)]+3 v
\end{aligned}
$$

for $d=3$ lattices with a trap at a corner site.

It is important to stress that the right-hand side of each of the above expressions is an integer. Hence, calculating the $\langle n(i)\rangle$ in integer format, constructing $\sum(1)$, and $\sum(2)$, and comparing the results obtained with the (integer) results predicted by the right-hand side of the exact formulae above confirms (or not) the numerical accuracy of our calculations. Table 3 gives the results for $\sum(1)$, and $\sum(2)$ for all the lattices considered in this study and provides the desired confirmation.

\section{Conclusions}

In this contribution we have studied ergodic flows of a random walker undergoing unbiased displacements in a positional phase space represented by a host lattice, and characterized quantitatively the consequences of different metric decompositions of a given, finite parent lattice. As a model for studying the early stages of self-assembly of nanoparticles, the results here complement those reported in $[3,4]$ where the phase (reaction) space was defined by a host, periodic lattice. Specifically, by breaking the translational symmetry of the reaction space, we find that the reaction efficiency in a finite system is strongly dependent not only on whether the system is compartmentalized, but also on whether the overall reaction space of the microreactor is further decoupled into separable reactors.

Finally, the influence of system dimensionality on the efficiency of kinetic processes carried out in compartmentalized microreactors is quantified. We find that more significant differences are encountered when a $d=2$ reaction space is deconstructed than a $d=3$ reaction space. Phrased in the language of experimental studies on nanoparticle kinetics, our results suggest that reactions carried out on articulated surfaces of a compartmentalized nanoparticle are likely to influence the efficiency of reaction to a greater extent than if the same reaction occurs in the partitioned interior of a $d=3$ nanoassembly.

\section{Acknowledgments}

One of the authors (J. J. Kozak) gratefully appreciates conversations with and the technical assistance of Amelia E. Pawlak, DePaul University. The work of Professor R. A. Garza-López was supported by the Hirsh Research Initiation Grant, the Howard Hughes Medical Institute Research Program, and the Summer Undergraduate Research Program from the Pomona College.

\section{References}

[1] A. N. Goldstein, Handbook of Nanophase Materials, Dekker, New York, NY, USA, 1977.

[2] P. Jensen, "Growth of nanostructures by cluster deposition : a review," Reviews of Modern Physics, vol. 71, no. 5, pp. 16951735, 1999.

[3] J. J. Kozak, C. Nicolis, and G. Nicolis, "Modeling the early stages of self-assembly in nanophase materials," Journal of Chemical Physics, vol. 126, no. 15, Article ID 154701, 2007.

[4] J. J. Kozak and G. Nicolis, "Modeling the early stages of selfassembly in nanophase materials. II. Role of symmetry and dimensionality," Journal of Chemical Physics, vol. 134, no. 6, Article ID 064701, 8 pages, 2011.

[5] J. J. Kozak, "Chemical reactions and reaction efficiency in compartmentalized systems," Advances in Chemical Physics, vol. 115, pp. 245-406, 2000.

[6] R. A. Garza-López, P. Bouchard, G. Nicolis, M. Sleutel, J. Brzezinski, and J. J. Kozak, "Kinetics of docking in postnucleation stages of self-assembly," Journal of Chemical Physics, vol. 128, no. 11, Article ID 114701, 2008.

[7] E. W. Montroll and G. H. Weiss, "Random walks on lattices. II," Journal of Mathematical Physics, vol. 6, no. 2, pp. 167-181, 1965.

[8] R. A. Garza-López and J. J. Kozak, "Invariance relations for random walks on hexagonal lattices," Chemical Physics Letters, vol. 371, no. 3-4, pp. 365-370, 2003.

[9] R. A. Garza-López and J. J. Kozak, "Invariance relations for random walks on square-planar lattices," Chemical Physics Letters, vol. 406, no. 1-3, pp. 38-43, 2005.

[10] R. A. Garza-López, A. Linares, A. Yoo, G. Evans, and J. J. Kozak, "Invariance relations for random walks on simple cubic lattices," Chemical Physics Letters, vol. 421, no. 1-3, pp. 287294, 2006. 


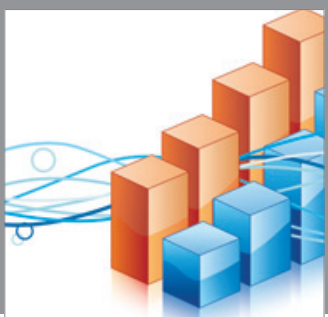

Advances in

Operations Research

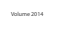

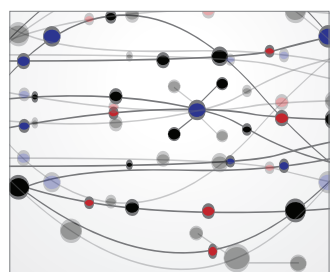

\section{The Scientific} World Journal
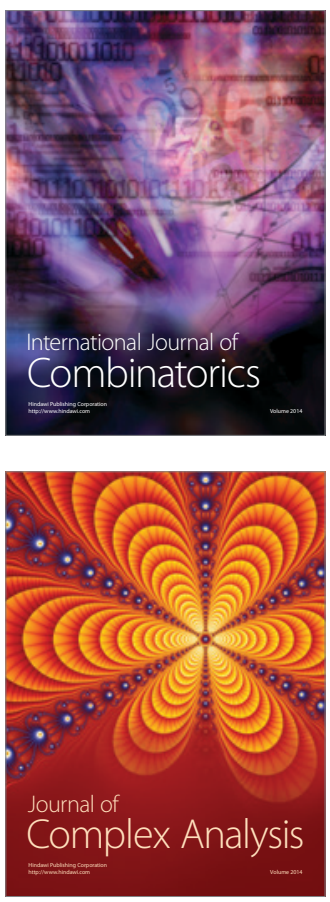

International Journal of

Mathematics and

Mathematical

Sciences
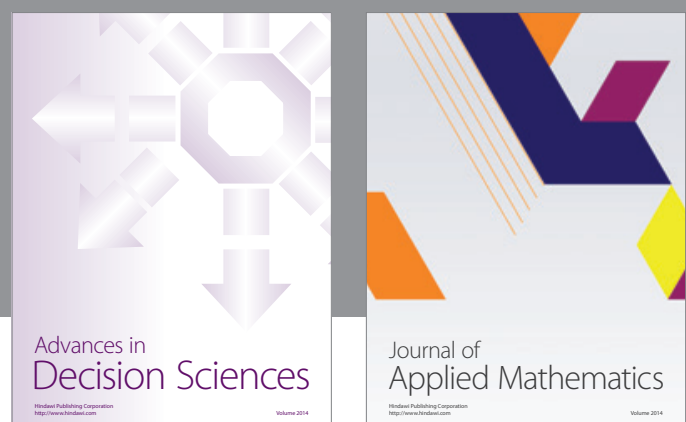

Journal of

Applied Mathematics
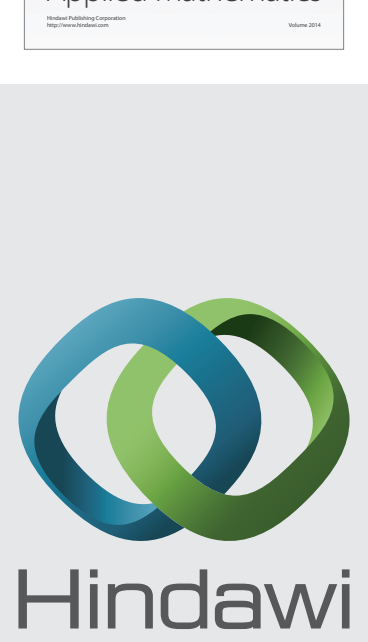

Submit your manuscripts at http://www.hindawi.com
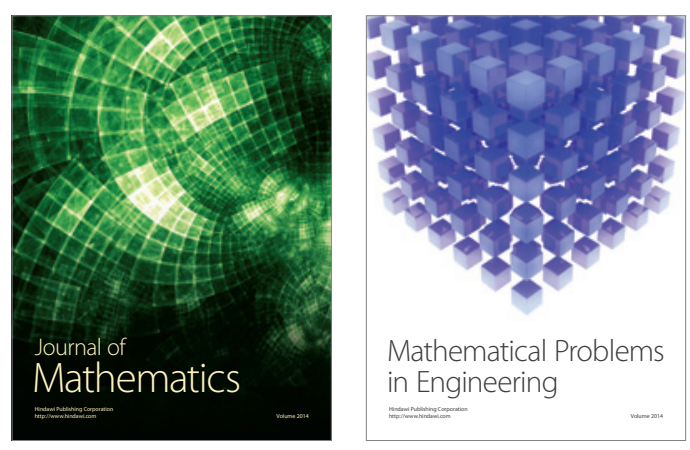

Mathematical Problems in Engineering
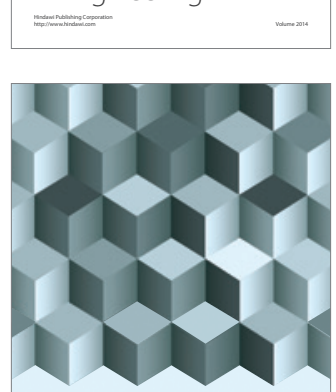

Journal of

Function Spaces
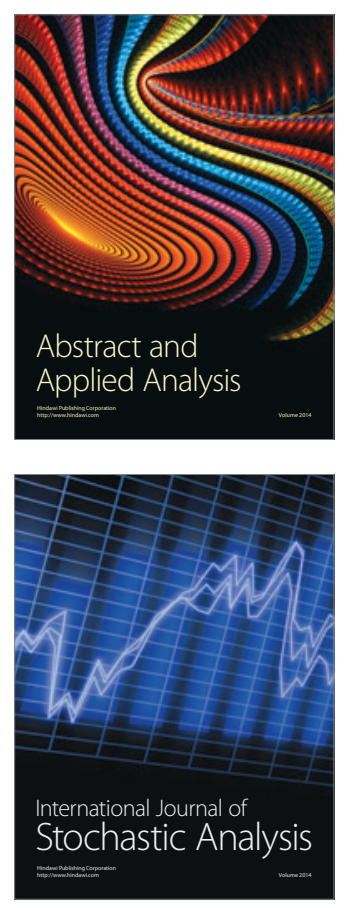

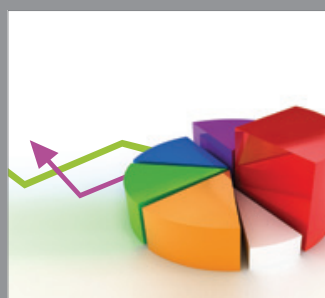

ournal of

Probability and Statistics

Promensencen
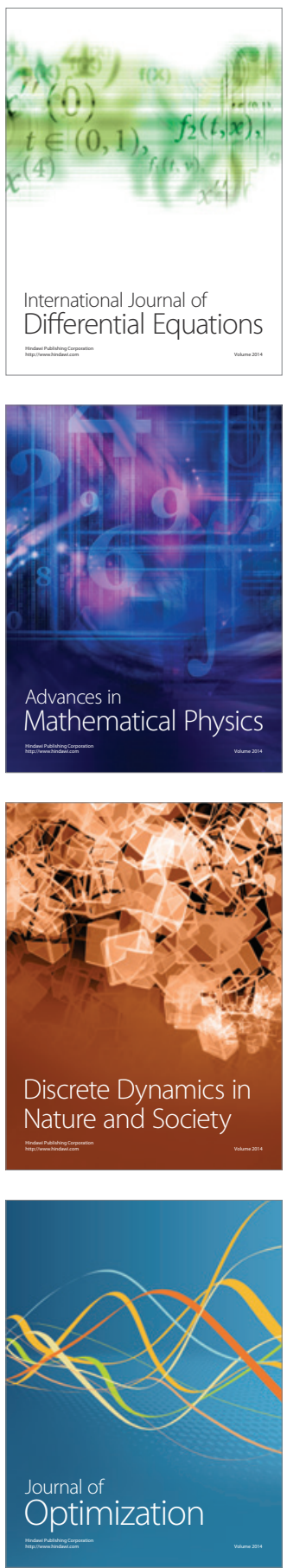